\title{
Emploi du vaccin avianisé souche B. A. contre la peste bovine en Afrique Centrale
}

\author{
par A. PROVOST, J. M. VILLEMOT $\dagger$ ef R. QUEVAL
}

\section{I. - NÉCESSITÉ D'UN NOUVEAU TYPE DE VACCIN ANTIPESTIQUE EN AFRIQUE CENTRALE}

Employé au Cameroun depuis 1945 (1) et au Tchad depuis 1947, d'abord sous forme de virus «humide» (2) puis de vaccin lyophilisé (3), le virus-vaccin capripestique a obtenu un beau succès auprès de ses utilisateurs. Il a permis de faire régresser d'une façon spectaculaire la peste bovine, qui ne se manifeste plus maintenant que parmi la population des jeunes veaux de l'année non encore vaccinés.

Longtemps, on a jugé la mortalité post-vaccinale, qui avoisine 2 p. 100, acceptable, bien que dans quelques troupeaux elle eût atteint $50 \mathrm{p}$. 100 ; on la mettait sur le compte d'hémoprotozooses ou d'helminthoses sous-jacentes, dont l'action pathogène se réveillait à la faveur de la vaccination.

Pourtant, quelques sondages discrets laissaient à penser au Laboratoire dès 1957, que le chiffre rassurant de 2 p. 100 se trouvait largement dépassé. U. i interrogatoire serré des éleveurs pris individuellement permettait de donner 20 p. 100 de mortalité chez les primo-vaccinés comme un chiffre plus près de la vérité. C'est ce pourcentage qu'obtient également PLOWRIGHT en Nigéria du Nord (4) sur une population de zébus Fulani, dans des conditions écologiques rappelant celles du Tchad et du Nord-Cameroun.

II fallait donc songer à remplacer dans l'avenir le vaccin capripestique par un autre vaccin de valeur antigénique comparable mais pius atténué, sans pour cela faire retour au vaccin formolé saponiné, dont la production était rendue difficile par le trop petit nombre de veaux réagissants à l'inoculation virulente et dont l'immunité

Reçu pour publication : octobre 1961.

Rev. Elev. Méd. vét. Pays trop., 1961, 14, no 4. qu'il conférait ne remplissait pas les conditions acceptables d'une politique de vaccination visant à l'éradication de la maladie.

La production de vaccin lapinisé ne pouvait être envisagée elle non plus faute de lapins au Tchad, l'élevage de ce rongeur étant particulièrement malaisé en zone sahélienne. Par ailleurs, on devait se demander si la fragilité du produit lyophilisé ne serait pas un obstacle à sa diffusion sur le terrain, dans les conditions climatiques et pratiques du Tchad.

Pour ces différentes raisons, nous nous sommes tournés vers l'étude, pour une utilisation future, des vaccins antibovipestiques avianisés. Après quelques tentatives infructueuses d'adaptation du virus capripestique à l'œuf embryonné, nous avons décidé de choisir l'une des souches déjà étudiées par d'autres laboratoires.

Au début de 1958, existaient les souches:

- DAUBNEY (5), dont on n'a malheureusement que peu d'expérience (6) ;

- B. A., de ISHII et TSUKUDA (7), dont l'étude expérimentale venait d'être entreprise à $\mathrm{Mu}$ guga (8) et dont les premiers résultats semblaient prometteurs :

- L. A., de NAKAMURA el MIYAMATO (9), dont on connaissait quelques-unes des caractéristiques (10).

Les deux dernières souches (BA ef $L A$ ) furent retenues et furent importées du KENYA en mars $1957\left(^{*}\right)$. Aucune difficulté ne fut rencontrée pour les passages de la souche BA en œuf de poule embryonné, et l'étude et la production du vaccin purent être menées à bien. Par contre, malgré plusieurs tentatives, la souche LA ne put être repassée dans l'œuf. La même observation a été faite à Vom par J. NAKAMURA lui-même (11)

(*) II nous est particulièrement agréable de remercier ici notre ami G. R. SCOTT de I'E. A. V. R. O.. Muguga, Kenya, qui a eu l'obligeance de nous envoyer ces souches. 
ainsi qu'à Dakar (12). Des facteurs encore inconnus doivent intervenir dans la multiplication du virus dans l'œuf de poule, tout au moins en Afrique ; une communication récente (13) ne les a pas tous élucidés.

\section{II. - PRODUCTION DU VACCIN AVIANISÉ B. A.}

\section{I. - Entretien de la souche}

On utilise des œufs de poule embryonnés de 7 jours, autant que possible à coquille blanche. Les cufs que nous utilisons sont importés de France car les poules de notre élevage étant nourries avec de la viande pestique, on peut craindre qu'une élaboration éventuelle d'anticorps antipestiques par l'organisme des volailles ait une influence défavorable sur la propagation in ovo du virus.

L'inoculum est le passage précédent, dilué à parties égales avec du bouillon ordinaire à pH7,2 à seule fin de rendre le liquide plus fluide et de faciliter les inoculations.

Les inoculations sont réalisées par voie vitelline en injectant directement $0,25 \mathrm{ml}$ de l'inoculum dans le jaune. Les œufs sont remis à incuber à $35^{\circ}$. Un contrôle des mortalités non spécifiques dues au traumatisme de l'inoculation est effectué 48 heures plus tard.

La récolte s'effectue le $8 \mathrm{e}$ jour suivant l'inoculation. On ne recueille que les œufs encore vivants. Toutefois, on doit faire le plus grand cas des œufs dans lesquels on trouve morts des embryons bien formés (c'est-à-dire des embryons qui sont morts au 15 ou $16 \mathrm{e}$ jour d'incubation). En pratique, on ne retient que les récoltes pour lesquelles on trouve 40 p. 100 des embryons morts le $8 \mathrm{e}$ jour après l'inoculation.

En effet, ISOGAI, ISHII, KATAOKA et FUKUSHO (16) d'une part, PIERCY, SCOTT et WITCOMB (14) d'autre part, ont remarqué que 9 jours après l'inoculation, 70 à 90 p. 100 des embryons inoculés étaient morts. Nous avons également vérifié ce fait. En récoltant le $8 \mathrm{e}$ jour, on ne doit trouver qu'environ 40 p. 100 de mortalité ; ce pourcentage laisse présumer une bonne multiplication viralc qu'il serait difficile de vérifier autrement que par l'inoculation des bouvillons sensibles ou la mise en œuvre de la technique de déviation du complément de J. NAKAMURA (15), ce qui introduirait une complication certaine pour les passages de routine. Nous nous sommes tenus avec succès à cette simple épreuve de lecture de mortalifé lors des récoltes.

Les récoltes sont effectuées sur l'œuf entier. Un broyage soigné au mixer et l'addition d'antibiotiques (Pénicilline : $1.000 \mathrm{U} / \mathrm{ml}$, Streptomycine $100 \mu \mathrm{g} / \mathrm{ml}$ ) complètent l'opération. Le produit est réparti en parties aliquotes et conservé congelé au réfrigérateur à $-20^{\circ} \mathrm{C}$.

\section{2. - Production du vaccin}

Un lot de production comprend de 100 à 200 œufs inoculés, quantité maxima imposée par la capacité de l'appareil à lyophiliser. Les inoculations ef la réincubation prennent place comme il a été indiqué plus haut.

Toutefois, les récoltes sont effcctuées le $7 e$ jour, de façon à recueillir un plus grand nombre d'embryons vivants. Il est courant de trouver déjà une mortalité de 10 à 15 p. 100.

Les récoltes sont broyées, additionnées d'antibiotiques, homogénéisées, puis diluées à parties égales dans notre milieu standard pour lyophilisation (3) inspiré du tampon de Fry. I'e vaccin frais est réparti à raison de $5,5 \mathrm{ml}$ de produit par flacon type pénicilline de $20 \mathrm{~cm}^{3}$, puis congelé à $-50^{\circ} \mathrm{C}$ sur les plateaux condenseurs de l'appareil à lyophiliser Stokes (modèle 2J05-FFX-4), les flacons étant couchés pour augmenter la surface d'évaporation lors de la mise sous vide.

Après congélation, on procède à un cycle de lyophilisation de 24 heures, à la suite duquel les flacons sont bouchés sous vide, capsulés, puis stockés à $-20^{\circ} \mathrm{C}$ en attendant leur titrage ef leur distribution.

le vaccin se présente comme une galefte blancjaunatre, d'aspect très poreux pesant $0,5 \mathrm{~g}$; sa remise en suspension est particulièrement aisée.

\section{3. - Titrage}

Il est effectué sur des veaux Bororos sensibles à la peste que nous importons de République Centrafricaine, territoire où la peste bovine n'a 
pas sévi depuis 1947 ef où uucune vaccination antipestique n'est pratiquée.

Dans un premier temps, il convenait de rechercher la dose minima immunisante pour le bœuf. A cet effet, sont préparées avec $1 \mathrm{~g}$ de vaccin des dilutions de $10^{-1}$ à $10^{-7}$ ef $1 \mathrm{ml}$ de chaque dilution est inoculé par voie sous-cutanée, à raison de 2 veaux par dilution. L'épreuve virulente est réalisée trois semaines plus tard avec la souche bovipestique lyophilisée du laboratoire.

Aucune réaction thermique ni organique ne s'est manifestée chez les bovins d'expérience à l'épreuve virulente, les animaux vaccinés avec les dilutions $10^{-1}$ à $10^{-5}$ y compris résistent, tandis que ceux ayant reçu la dilution $10^{-7}$, ainsi que des témoins, contractent la peste. Il est évident que $1 \mathrm{ml}$ de la dilution à $10^{-6}$ confère l'immunité. Ce résultat est en accord avec celui de PIERCY et WITCOMB $(8,17)$.

Les flacons de vaccin contiennent $0,5 \mathrm{~g}$ de produit sec ; en multipliant la dose minima immunisante par 250, on doit se mettre à l'abri de toute perte de virus pour le travail sur le terrain; chaque flacon renferme donc 200 doses vaccinales.

Ce titrage a été réalisé pour les premiers lots de vaccin. Par la suite, on s'est contenté de rechercher pour chaque lot produit si la dose vaccinale pratique $(1 \mathrm{ml}$ de la dilution au $1 / 200 \mathrm{e}$ du vaccin contenu dans un flacon) conférait l'immunité.

\section{III. - UTILISATION SUR LE TERRAIN}

Nous en étions à ce stade de l'étude du vaccin avianisé lorsque de grosses demandes nous furent faites simultanément par les services de l'Elevage du Tchad et du Cameroun. Ayant fait toutes les réserves qui s'imposaient pour une utilisation à grande échelle de ce vaccin, son emploi fut néanmoins décidé pour les raisons que l'on verra plus loin.

II convient, pour plus de clarté, d'envisager l'utilisation du vaccin avianisé en zone d'épizootie pestique, puis en zone d'enzootie, pour mettre en lumière quelques faits saillants de son pouvoir immunogène.

\section{A. - UTILISATION EN ZONE D'ÉPIZOOTIE PESTIQUE}

\section{Schéma épizootologique et choix du vaccin.}

Pour des raisons restćcs cncore mystérieuses, la peste bovine a éclaté brusquement en avril 1960, près de Tibati, dans l'Adamaoua (Cameroun central). Malgré des mesures de prophylaxie sanitaire aussitôt mises en place (abattage des troupeaux malades) la maladie gagnait très vite. En effet, l'Adamaoua était jusque là, sur le plan de l'épizootologie bovipestique, une région privilégiée : la dernière épizootie de peste remonte à 1927 ; elle fut d'ailleurs catastrophique, luant les $2 / 3$ du troupeau.

Depuis lors, entouré de zones à forte infestation trypanosomienne interdisant le passage des troupeaux transhumants, l'Adamaoua était resté indemne et aucune vaccination anfipestique n'y était pratiquée. C'est dire que la sensibilité des zébus peuhl à la peste était entière. En effet, dans les premiers troupeaux atteints; la morbidité fut de 100 p. 100 et la mortalité de plus de 90. L'isolement sanitaire des foyers était rendue illusoire par suite du manque de coopération des éleveurs qui, ignorant ce qu'était la peste et peu effrayés au début par sa nature contagicusc, sc prêtaient difficilement à des mesures sanitaires. L'incompréhension des commerçants en bétail aida pour une part à la dissémination du contage, qui eut également en quelques occasions le gibier pour le colporter. C'est pourquoi, en dehors de mesures sanitaires extrêmement précaires, les armes mises à la disposition du service de l'Elevage du Cameroun étaient les suivantes:

- le vaccin formolé, difficile à produire sur place et dont on ne pouvait faire guère plus de 8.000 doses par jour, alors qu'il y avait 600.00 bovins à vacciner le plus vite possible.

- Le vaccin capripestique, qui a fait la preuve de son efficacité mais dont on pouvait craindre que l'utilisation sur ces animaux très sensibles ne donnât des réactions vaccinales fâcheuses. C'est ce que montra d'ailleurs une expérience pilote : sur 10 veaux vaccinés, trois moururent de réaction vaccinales aiguës extrêmement violentes, tandis que les sept autres étaient fortement toui chés. 
TABLEAJ II - Mortalités dues à la peste ou à ses séquelles sur des troupeaux dans lesouels la maladie a évolué moins de 12 jours après ia vaccination.

\begin{tabular}{|c|c|c|}
\hline \multirow{2}{*}{$\begin{array}{c}\text { Effectif } \\
\text { avant la peste }\end{array}$} & \multicolumn{2}{|c|}{ Mortalité } \\
\cline { 2 - 3 } & Nombre & Pourcentage \\
\hline 100 & 35 & 35 \\
50 & 24 & 48 \\
64 & 45 & 41,5 \\
74 & 17 & 23 \\
76 & 31 & 40 \\
108 & 34 & 31,5 \\
163 & 60 & 30,7 \\
42 & 24 & 57,1 \\
20 & 3 & 15 \\
26 & 2 & 7 \\
70 & 30 & 42,5 \\
28 & 9 & 32 \\
\hline \multicolumn{2}{|l}{} \\
\end{tabular}

TABLEAU I - Mortalités dues à la peste bovine dans des troupeaux non vaccinés.

\begin{tabular}{|c|c|c|}
\hline \multirow{2}{*}{$\begin{array}{c}\text { Effectif } \\
\text { du troupeau }\end{array}$} & \multicolumn{2}{|c|}{ Nortalité } \\
\cline { 2 - 3 } & Nombre & Pourcentage \\
\hline 100 & 100 & 100 \\
546 & 456 & 86 \\
149 & 123 & 83,5 \\
423 & 287 & 68 \\
60 & 50 & 83,3 \\
52 & 43 & 83 \\
\hline & & 80 \\
\hline
\end{tabular}

TABLEAi III - Hortalités dues à la peste ou à ses séquelles sur des troupeaux dans lesquels la maladie a évolué plus de 12 jours après la vaccination.

\begin{tabular}{|c|c|c|c|c|}
\hline \multirow{2}{*}{$\begin{array}{c}\text { Date } \\
\text { de vaccination }\end{array}$} & \multirow{2}{*}{$\begin{array}{l}\text { Apparition } \\
\text { de la maladio }\end{array}$} & \multirow{2}{*}{$\begin{array}{l}\text { Effectif } \\
\text { vacciné }\end{array}$} & \multicolumn{2}{|c|}{ Mortalité } \\
\hline & & & Nombre & Pourcentafe \\
\hline $17-5-60$ & $10-6-60$ & 28 & $15:$ & 53 \\
\hline $18-5-60$ & $10-6-60$ & 21 & 3 & 14 \\
\hline $18-5-60$ & $15-6-60$ & 29 & 1 & 3,5 \\
\hline $18-5-60$ & $15-6-60$ & 134 & 4 & 3 \\
\hline $18-5-60$ & $15-6-60$ & 28 & 5 & 18 \\
\hline $22-5-60$ & $16-6-60$ & 70 & 3 & 4 \\
\hline $21-5-60$ & $16-6-60$ & 20 & 3 & 15 \\
\hline $27-5-60$ & $18-6-60$ & 77 & 2 & 2,5 \\
\hline $19-5-60$ & $16-6-60$ & 200 & 70 & 35 \\
\hline $19-5-60$ & $16-6-60$ & 60 & 5 & 8 \\
\hline $19-5-60$ & $16-6-60$ & 200 & 15 & 7,5 \\
\hline $18-5-60$ & $16-6-60$ & 42 & $\begin{array}{l}5 \text { malades } \\
\text { guéris }\end{array}$ & 0 \\
\hline $20-5-60$ & $5-7-60$ & 37 & 3 & 8 \\
\hline \multicolumn{5}{|c|}{ Moyerine } \\
\hline
\end{tabular}


- Le vaccin lapinisé qui n'est pas produit par le Laboratoire de Farcha pour les raisons invoquées plus haut.

- Restait le vaccin avianisé dont nous avions 400.000 doses en stock. La vaccination généralisée du troupeau de l'Adamaoua fut décidée (600.000 têtes environ) après qu'une autre expérience pilote eût montré la totale innocuité de ce vaccin pour ce type de bétail : 10 veaux vaccinés avec 1 dose vaccinale de vaccin avianisé ne montraient aucune réaction clinique ni thermique : ils résistaient à l'inoculation virulente effectuée 9 et 10 jours après la vaccination. Toutefois, trois présentaient un peu de larmoiement, mais sans perdre l'appétit. Ce dernier fait est d'importance.

\section{Vaccination.}

La vaccination à l'aide de ce vaccin fut faite en réalisant d'abord autour des foyers, un solide cordon de protection à partir duquel rayonna la vaccination. La campagne fut réalisée dans des conditions techniques remarquables, par des équipes parfaitement entraînées; à aucun moment la chaîne du froid n'a été rompue et le vaccin a été employé sur le terrain dans des conditions très bonnes.

\section{Résultats.}

L'influence de la vaccination a été nette: les chiffres de 80 p. 100 de morts et de 20 p. 100 de survivants dans les troupeaux non vaccinés touchés par la vague épizootique se sont trouvés inversés. Toutefois ce qui semble important, c'est qu'un certain nombre d'animaux ont contracté la peste.

Les éleveurs se sont déclarés satisfaits, mais la vaccination seule n'a pas limité l'extension de l'épizootie ; elle a fait apparaître chez les vaccinés une certaine immunité antipestique, totale pour près de 80 p. 100 d'entre eux, subtotale pour 10 p. 100 , inexistante pour le restant. Cette proportion se retrouve dans un même troupeau, vacciné avec le même lot de vaccin, parfois avec le même flacon, dans des conditions identiques.

Les tableaux I, II et III, rendent respectivement compte de l'évolution de la maladie dans quelques troupeaux non vaccinés, dans des trou- peaux vaccinés depuis moins de 12 jours et dans des troupeaux vaccinés depuis plus de 12 jours.

II est certain que dans le $2 \mathbf{e}$ groupe d'animaux un certain nombre d'entre eux étaient soit en incubation de peste, soit ont été contaminés dans les tout premiers jours suivant la vaccination. II ne semble pas que le phénomène d'interférence, qui existe avec le vaccin capripestique ef le vaccin lapinisé, ait joué en ces circonstances. Nous n'avons malheureusement pu vérifier ce fait d'une manière plus rationnelle que par l'observation de l'épizootologie de la maladie.

Parmi les troupeaux vaccinés depuis plus de 12 jours, le degré d'immunité antipestique est augmenté sans qu'il soit pour autant total. On constate un fait curieux pour un vaccin à virus vivant atténué : c'est que, bien qu'il ait été inoculé dans des conditions similaires à des bovins sans passé immunologique antipestique, la « loi du tout ou rien " n'ait pas joué ; on constate dans un même troupeau tous les degrés de la susceptibilité à la maladie, de la résistance totale à la sensibilité complète, sans que semblent intervenir des facteurs comme l'âge, le sexe, ou l'état nutritionnel des vaccinés.

Il est à remarquer que les résultats de cette large enquête, effectuée sur plusieurs centaines de milliers de bovins, recoupe l'expériencepilote que nous avons évoquée plus haut : à l'inoculation virulente, trois veaux sur dix avaient réagi par un peu de larmoiement, symptôme fruste de peste bovine qui n'a pas semblé alarmant sur le champ mais qui a posteriori se montre riche d'enseignement.

Un titrage des anticorps neutralisants, selon la technique de SCOTT et BROWN (18), effectué sur les sérums de zébus vaccinés depuis un mois, a fourni des titres neutralisants de $10^{-1}$ au maximum. C'est dire que l'on se trouve au seuil minimum du titre en anticorps neutralisants nécessaire pour protéger un bovin contre la peste.

II est d'ailleurs à remarquer que ce titre n'a pas varié dans les 10 mois suivant la vaccination ainsi que l'ont montré d'autres sondages sérologiques réalisés en mai 1961.

Ce résultat donne l'explication de la gradation des symptômes observés dans les troupeaux ayant contracté la peste après la vaccination, selon que la teneur en anticorps des vaccinés a dépassé ou a été inférieure au șeuil critique.

L'éclosion dans des troupeaux, vaccinés, de 
foyers secondaires dus au colportage du virus par des animaux de boucherie, imposa au service vétérinaire du Cameroun le recours au vaccin formolé. Son emploi (55.000 vaccinations effectuées en cordon sanitaire autour des foyers secondaires) devait permettre la disparition de la maladie. Depuis 9 mois, tout semble calme à ce point de vue dans l'Adamaoua.

\section{B. - UTILISATION EN ZONE D'ENZOOTIE PESTIQUe}

Dans le même temps que la vaccination généralisée de l'Adamaoua étnit mise en œuvre, des campagnes de vaccination avec le vaccin avianisé étaient établies dans les régions du MayoKebbi (sud-ovest du Tchad) et du Nord-Cameroun. Tout l'intérêt de ce type de vaccin venait de sa possibilité d'emploi, sans réactions fâcheuses, sur un bétail trypanosomé latent.

Dans ces provinces, l'épizootologie de la peste est analogue à ce qu'elle est dans le reste de l'Afrique sahélienne; elle y subsiste à l'état enzootique, touchant principalement les jeunes non encore vaccinés. C'est sur eux qu'à porté l'effort de vaccination dans ces campagnes. L'état immunitaire antipestique de ces animaux « tout venant » était inconnu, et il étcit possible qu'une certaine proportion d'entrc cux hébergeât encore des anticorps neutralisants d'origine maternelle, reliquat șuffisant pour neutraliser le vaccin injecté et compromettre ainsi le stimulus antigénique.

180.000 vaccinations ont été réalisées dans ces conditions. Aucune réaction clinique ni thermique n'a suivi la vaccination. Aucune poussée épizootique de peste ne s'est manifestée, mais par contre, certains troupeaux ont été touchés par suite de la pérennité du contage.

La marche de la maladie a été différente de ce qu'elle fut dans les troupecux « neufs » vaccinés de l'Adamaova. Dans le Nord-Cameroun notamment, on a pu constater que pratiquement tous les jeunes bovins vaccinés d'un troupeau faisaient la peste, mais qu'un nombre relativement restreint en mourait (10 p. 100 au plus). Les autres faisaient une peste peu grave.

Il est assez surprenant de constater que malgré ce défaut caractérisé de protection, la vaccination a joui de la faveur des éleveurs. Sans doute les perfes étaient-elles inférieures à celles constatées après l'emploi du capripestique.

\section{IV. - DISCUSSION}

L'innocuité du virus-vaccin BA ne saurait être mise en doute ' I'inoculation n'est suivie d'aucune réaction fâcheuse, d'aucune mortalité ; l'exacerbation d'une trypanosomiase latente ne semble pas à craindre.

Par contre, il semble souffrir de gros reproches sur le plan de son efficacité. Il convient d'en analyser les raisons.

La pauvreté de la réponse immunologique à l'inoculation peut-elle trouver une explication logique dans la trop grande atténuation de cette souche, qui, bien qu'inoculée à un bétail zébu hautement réceptif à la peste, semblerait ne pas devoir s'y multiplier de façon satisfaisante ? Une étude de la virémie à virus BA chez des zébus vaccinés eût été du plus haut intérêt; nous n'avons pu la mener à bien faute de bétail pleinement et sûrement sensible au laboratoire.

Un fait vient cependant à l'appui de cette carence de la multiplication virale : c'est l'absence d'interférence que I'on peut remarquer après la vaccination. Le mécanisme intime de l'interférence prête encore à l'hypothèse, soit que jove un blocage des récepteurs viriens des cellules sensibles au virus bovipestique, soit qu'une substance du type interféron (19), soit relarguée lors de l'infection cellulaire. Les deux théories supposent toutefois l'infection des cellules sensibles (dans le cas présent, celles du S. R. E.) par un nombre suffisant de particules infectieuses du virus «interférant », c'est-à-dire qu'il semble que doive avoir lieu au moins un cycle de reproduction de ce virus pour toucher un nombre suffisant de cellules. Si effectivement la reproduction du virus $B A$ ne s'effectue pas ou s'effectue difficilement chez le zébu, on tient la double explication de l'absence d'interférence et de la faiblesse de la réponse immunologique.

Il est à noter dans cet ordre d'idée, que le délai d'apparition du phénomène d'interférence avec les divers virus-vaccins antipestiques est un reflet de l'adaptation à un hôte hétérologue de plus en plus éloigné du bœuf et semble corrélatif de la précocité de la virémie après injection de ces virus au bœuf.

Cette hypothèse ne serait pas la première qui incriminerait l'excessive atténuation des virus pestiques avianisés. Le fait est survenu à Grosse- 
Isle dans les mains de l'équipe américano-canadienne (20) ainsi qu'au Kenya (21). Les passages de la souche BA, réalisés à Muguga puis à Farcha après son envoi du Japon, ont-ils pu réaliser ce surcroît d'atténuation ?

Cela semble difficile à admettre. La souche, à son 237 e passage en vitellus à son arrivée au Kenya, a été envoyée à Farcha d̀ son 246e passage ; elle était toujours à ce stade parfaitement immunogène (G. R. SCOTT, communication personnelle). Nous avons effectué cinq passages, et le vaccin employé en Adamaoua était donc le $251 \mathbf{e}$ passage. Il semble a priori improbable, étant donné le passage correct du virus in ovo, démontré par les mortalités embryonnaires, et les résultats heureux du titrage sur bœuf effectué au laboratoire, que le virus ait subi une brusque mutation de son pouvoir immunogène. Une autre cause doit être retenue.

II semble bien que des susceptibilités raciales soient en cause. En effet, dans les mains de PIERCY et WITCOMB $(8,17)$ le virus BA donnait des réactions thermiques $\left(2^{\circ} \mathrm{F}\right.$, soit $1,1^{\circ} \mathrm{C}$ ) sur le type de bétail qu'ils employaient (métis de races européennes et de races locales de l'Est africain). Or dans nos expériences de laboratoire et lors de l'application sur le terrain, jamais nous n'avons constaté la moindre élévation thermique

La réceptivité du bétail au virus bovipestique virulent étant mise hors de cause sur le plan immunologique, seule reste l'hypothèse de la susceptibilité raciale, avec tout ce que cela recèle d'ignorance de notre part.

Ce n'est pourtant point la première fois que nous suspectons cefte hyposensibilité des races bovines centrafricaines aux virus pestiques. Les titrages de virus bovipestique virulent et de virus capripestique, que nous réalisons de temps à autre sur du bétail bororo importé de R. C. A., montre des anomalies : tel animal, par exemple, inoculé avec une dilution sûrement infectante de virus $\left(10^{-1}\right.$ ou $\left.1^{-3}\right)$ ne réagira absolument pas, bien que son origine et un contrôle sérologique préalable aient permis de le supposer entièrement réceptif. De plus, une observation clinique de l'épizootie de l'Adamaoua vient renforcer cette opinion.

Nous y avons noté l'extrême fréquence des formes cutanées de peste, dont on s'accorde à dire qu'elles se manifestent sur un bétail semirésistant ou qu'elles sont l'apanage des épizoo- ties peu graves. A l'inverse de races hyposensibles, on connaît des races hypersensibles, dont le bétail coréen est un exemple. L'explication rationnelle de ces différences de comportement n'a pas encore été fournie bien qu'elle doive résider dans le patrimoine génétique des cellules.

L'argumentation précédente nous conduit donc a énoncer que l'échec partiel de lá vaccination au vaccin avianisé souche BA doit tenir à l'hyposensibilité génétique (euphémisme qui cache notre ignorance) des races bovinies centrafricaines au virus pestique.

Sur ce type de bétail (où se côtoient les races arabes ou choa, bororos et peuhls), le virus avicnisé possède un «pouvoir d'attaque » insuffisant, une afténuation trop grande, pour pouvoir s'établir en des cycles de reproduction réguliers amenant une réponse immunologique appréciable et constante dans la population bovine vaccinée.

Quoique satisfaisant les éleveurs, et tout spécialement ceux vivant dans des zones d'endémicité trypanosomienne, le virus BA n'est pas un vaccin à utiliser en Afrique centrale. Certes, il ne tue aucun animal lors de la vaccination: certes, près de 90 p. 100 des vaccinés ne meurent pas de peste si jamais la maladie atteint le troupeau, mais il n'assure pas l'éradication de la peste et risque de l'entrenir dans une population semi-immune. Imposant une fausse sûreté, déformant l'expression clinique classique de la peste et nuisant par là à la mise en place de mesures sanitaires, la vaccination au virus BA (comme celle mettont en cuvre tout autre virus-vaccin trop atténué) ne peut qu'être une gêne et impose le recours à d'autres méthodes de prophylaxie médicale.

C'est déjà ce que prévoyait RECEVEUR en 1952 (22), si l'usage de virus-vaccins trop atténués se répandait.

C'est pourquoi, malgré les demandes de ce type de vaccin, nous en avons arrêté la fabrication et la distribution. S'est reposé alors le problème d'un vaccin plus atténué que le vaccin capripestique ; la réponse semble être apportée par le vaccin de cultures cellulaires.

II est vraisemblable que les conclusions que nous venons de tirer ne sont vraies que pour l'Afrique Centrale.

Sur un autre type de bétail, et très vraisemblablement sur un bétail de type européen, l'immu- 
nité serait de qualité bien supérieure à celle que nous avons observée. L'étude du virus pestique avianisé BA mérite donc d'être poursuivie ailleurs, car il possède de réels avantages dont la facilité de production, le prix modique et les qualités de conservation. II pourrait être éventuellement la solution du problème de la vaccination du buffle du sud-est asiatique.

Institut d'élevage ef de médecine vétérinaire des pays tropicaux : Laborotoire de recherches vétérinaires de Farcha, Fort-Lamy (Tchad).

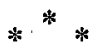

C'est pour nous un devoir et un plaisir de rendre hommage à nos confrères R. BLANC, R. DIDIERJEAN et R. FERNAGUT, du Service de I'Elevage du Cameroun ; A. LECLERCQ et D. ANNETT, du Service de l'Elevage du Tchad, pour l'aide précieuse qu'ils nous ont apportée en colligeant les résultats et en nous faisant part de leurs observations. C'est grâce à leur critique éclairée que nous avons connu l'étrangeté du comportement du virus BA.

\section{RÉSUMÉ}

Après avoir indiqué les raisons qui incitèrent à remplacer le vaccin capripestique par un vaccin plus atténué, les auteurs décrivent la production et le titrage du vaccin avianisé souche BA de ISHill et TSUKUDA. Ils en relatent l'emploi sur 800.000 bovins de l'Afrique Centrale.

Ils en tirent la conclusion qu'en Afrique Centrale tout au moins, la souche BA est un mauvais vaccin parce que trop atténuée pour le type de zébu semi-résistant à la peste bovine qué l'on y rencontre.

\section{SUMMARY}

The use of avianised-bovine virus rinderpest vaccine in the Central African Republic (West Africa)

Having explained the reasons for requiring in this country a more attenuated rinderpest vaccine than the caprinised type, the authors described the methods used to produce rinderpest vaccine of B. A. type Ishii and Tsukuda and the results obtained when vaccinating 800,000 head in this republic.

They reach the conclusion that this vaccine is of little value in the Central African Republic at least, since it is too attenuated to develop an immunological reaction in the rinderpest semi-resistant bovine breeds found there.

\section{RESUMEN}

\section{Empleo de vacuna avinizada cepa B.A. contra la peste bovina en Africa Central}

Despues de justificar las razones que incitaron a reèmplazar la vacuna capripestica por una vacuna mas atenuada, los autores describen la produccion y tifulación de la vacuna avinizada cepa B. A. de Ishii y Tsukuda.

Ellos la han empleado sobre 800.000 bovinos del Africa Central.

Concluyen que en Africa Central algunas veces la cepa B. A. es una mala vacuna por estar demasiado atenuada para el tipo de zebú, semi-resistente a la peste bovina que alli se encuentra. 


\section{BIBLIOGRAPHIE}

1. BLANC (R.). - La lutte contre la peste bovine au Cameroun. Bull. Acad. vét. France. $1947,20: 287$

2. SACQUET (R.) et TROQUEREAU (P.). Essai de vaccination contre la peste bovine au moyen du virus capripestique dans le nord-est du Tchad. Rev. Elev. Méd. vét. Pays trop., 1951-52, $5: 45$.

3. PROVOST (A), VILLEMOT (J. M.) et QUEVAL (R.). - La production du virus capripestique au laboratoire de Farcha. Bull. epiz. Dis. Afr., 1958, $6: 351$.

4. PLOWRIGHT (W). - Recent observations on rinderpest immunization and vaccines in northern Nigeria. Brit. vet. J., 1957, 113 : 385.

5. DAUBNEY (R.). - Peste bovine ; note sur les,vaccinations par virus vivants.Bull. O.I.E., 1951, $36: 116$

6. Kenya Veterinary Department. - Annual Report 1956, p. 26.

7. ISHII (S.) et TSUKUDA (K.). - Studies on the adaptation of bovine strain of rinderpest virus in chick embryo. Report Gov. Exp. Stap. an. Hyg., 1952, no 25, 29.

8. PIERCY (S. E.) et WITCOMB (M. A.). Studies on a japanese avianized strain on rinderpest virus. E. A. V.R. O. Annual Report 1954-55, p. 30.

9. NAKAMURA (J.) et MIYAMOTO (T.). Avianization of lapinized rinderpest virus. Am. J. vet. Res., 1953, $14: 307$.

10. NAKAMURA (J.), KISHI (S.) et MIYAMOTO (T.). - Sur les caracéristiques de la multiplication du virus lapinisé-avianisé de la peste bovine dans les embryons de poulet. Bull. O. I. E., 1954, $42: 692$.

11. NAKAMURA (J.). - Report to the government of Nigeria on production of rinderpest vaccine in northern Nigeria. F. A. O. Rapport no 768/1957.

12. Rapport annuel du Laboratoire Fédéral de l'Elevage Dakar-Hann, 1958.

13. MAC LEOD (A. J.) et KISHI (S.). - Some factors influencing the propagation of rinderpest virus in embryonated eggs. J. comp. Path., 1961, 7I : 140.

14. PIERCY (S. E.), SCOTT (G. R.) et WIT$\operatorname{COMB}(M$. A.). - Studies on avianized Rinderpest Virus in the Embryonated Egg. E. A. V. R. O. Annual Report, 1956-57, p. 17.

15. NAKAMURA (J.). - Complement fixation reaction in rinderpest study. Monographie de I'O. I. E. Paris, 1958.

16. ISOGAI (S.), ISHII (S.), KATAOKA (T.) et FUKUSHO (K.). - Behaviour of multiplication of avianized rinderpest virus in embryonating hen's eggs. Bull. not. Inst. anim. Health, Tokyo, 1959, $37: 147$.

17. PIERCY (S. E.), WITCOMB (M. A.). - Laboratory trials wiht an avianized rinderpest vaccine. Brit, vet. J., 1957, II3 : 353.

18. SCOTT (G. R.) ef BROWN (R.). - A neutralization test for the detection of rinderpest antibodies. J. comp. Path., 1958, $68: 308$.

19. ISAACS (A.) et LINDEWMANN (J.). - Virus interference. 1 . the interferon. Proced. Roy. Soc., 1957, B 147 : 258

20. HALE (M. W.) et WALKER (R. V. L.). Rinderpest. XIII. The production of rinderpest vaccine from an attenuated strain of virus. Am. J. vet. Res., 1946, 7 : 199.

21. HUDSON (J. R.). - Rinderpest virus attenuated in eggs. Vet. Rec., 1947, $59: 331$.

22. RECEVEUR (P.). - Réflexions sur l'épidémiologie de la peste bovine en Afrique Centrale. Bull. O. I. E., 1952, $37: 536$. 